\title{
ALGUNOS FACTORES QUE CONDICIONAN LA AUTOMEDICACIÓN EN ESTUDIANTES DE LA FACULTAD DE ENFERMERÍA DE LA UNIVERSIDAD NACIONAL SAN LUIS GONZAGA DE ICA, OCTUBRE 2011 - SETIEMBRE 2012.
}

Some factors that influence the self-medication in students from the faculty of nursing at the national university san luis gonzaga of ica, october 2011 - september 2012.

Isabel Natividad Urure Velazco ${ }^{1,2, a, b, e}$, Rosario Campos Soto ${ }^{1,2, a, c, d}$, Rosa Ramos Ramos ${ }^{1,3}$, Mirsa Arotuma Nieto ${ }^{4}$, Henry Bernaola Bello ${ }^{4}$, Melissa F. Canales Poma ${ }^{4}$.

${ }^{1}$ Facultad de Enfermería. Universidad Nacional San Luis Gonzaga de Ica. Ica, Perú.

${ }^{2}$ Hospital Santa María del Socorro. Ica, Perú

${ }^{3}$ Hospital Regional de Ica. Ica, Perú

${ }^{4}$ Estudiante de Enfermería. Universidad Nacional San Luis Gonzaga de Ica. Ica, Perú.

${ }^{a}$ Lic. en Enfermería. ${ }^{b}$ Enfermera Especialista en Cirugía General. $\quad{ }^{c}$ Enfermera Especialista en Cuidado del Niño.

${ }^{d}$ Mag. En Salud Pública. ${ }^{e}$ Doctor en Ciencias de la Salud.

\section{RESUMEN}

La automedicación es una práctica muy frecuente en nuestro medio y en el mundo entero, llevado a cabo por razones sociales, económicas y culturales, que muchas veces más que satisfacer las necesidades de salud, empeoran o agravan. Objetivo: Determinar cuáles son los factores que condicionan la automedicación en estudiantes de la Facultad de Enfermería de la Universidad Nacional San Luis Gonzaga de Ica. Material y métodos: Estudio descriptivo - transversal. Constituida por 136 estudiantes del I al IX Ciclo. Haciéndose uso de un cuestionario validado de 13 reactivos de opción múltiple y prueba estadística de chi cuadrado $\left(\mathrm{x}^{2}\right)$. Resultados: Factores sociales (entorno social): El farmacéutico y la familia recomendaron prioritariamente el medicamento el $52,9 \%$ y $30,9 \%$ respectivamente. Factores culturales: el $82,4 \%$ definieron acertadamente la automedicación como "uso de los medicamentos sin prescripción médica", el 81.6\% señalaron que tiene efectos negativos para la salud. El 39,0\% consulta al médico cuando está enfermo, siendo la televisión, el medio de comunicación que más influye en la automedicación 74.3\%. Factores Económicos: El 89\% obtiene el medicamento en farmacia, destinándose dinero del ingreso económico familiar para acceder a un servicio de salud el 52,2\%, cuyo monto económico mensual familiar es mayor de 750,00 Nuevos soles el 58,8\%. Se automedica 33,8\% (46 estudiantes del total de la muestra). Motivo más frecuente de automedicación es por dolor de cabeza $28,7 \%$ y por fiebre $26,5 \%$. Los medicamentos más utilizados que conllevan a la automedicación son analgésicos y antiinflamatorios el $75.7 \%$, automedicándose en los últimos 6 meses el 35\%. Conclusiones: Los factores sociales, culturales y económicos condicionan la automedicación en estudiantes de la Facultad de Enfermería de la Universidad Nacional San Luis Gonzaga de Ica. PALABRAS CLAVE: Factores condicionantes, automedicación, estudiantes enfermería.

\section{SUMMARY}

Self-medication is a very common practice in our country and around the world, carried out for social, economic and cultural, that many times you meet health needs, worsen or exacerbate. Objectives: To determine the factors that influence self-medication among students of the Faculty of Nursing at the Universidad Nacional San Luis Gonzaga de Ica. Material and methods: A descriptive - cross. Consisting of 136 students from I to IX Cycle. Making use of a validated questionnaire of 13 multiple-choice test and chi square statistic (x2). Results: Social factors (social 
environment): The pharmacist and family medicine primarily recommended $52.9 \%$ and $30.9 \%$ respectively. Cultural factors: the $82.4 \%$ self-medication aptly defined as "use of drugs without a prescription", $81.6 \%$ said they have negative health effects. The $39.0 \%$ consulted the doctor when sick, with television, the medium that most influences the self-medication $74.3 \%$. Economic Factors: The $89 \%$ get the drug from pharmacy, targeting money from family income to access a health service $52.2 \%$ whose family monthly budget amount is greater than 750.00 soles $58.8 \%$. Selfmedicate $33.8 \%$ (46 students total sample). Most common reason is self-medication for headache and fever $28.7 \% 26.5 \%$. The most used drugs that lead to self-medication and anti-inflammatory painkillers are $75.7 \%$, self-medication in the last 6 months to $35 \%$. Conclusions: The social, cultural and economic condition the students self-medication in students of the Faculty of Nursing at the Universidad Nacional San Luis Gonzaga de Ica.

KEYWORDS: Factors conditioning, self-medication, nursing students.

\section{INTRODUCCIÓN}

Según la organización Mundial de la Salud (OMS) la automedicación orientada es una forma de autocuidado a la salud. Profesionales calificados preferentemente el farmacéutico deben incentivar al uso racional de medicamentos, informar sobre los fármacos y las complicaciones que pueden originarse de su uso indiscriminado y si es necesario encaminar a las personas para atención médica, promoviendo la automedicación responsable (1), representando la automedicación en todos los países especialmente los que se encuentran en vías de desarrollo un gran problema de salud pública debido a los daños que a nivel de salud del individuo, la familia y la comunidad puede ocasionar (2).

En este sentido se puede señalar que la automedicación es una práctica muy frecuente en nuestro medio y el mundo entero, llevado a cabo por factores sociales, económicos y culturales muchas veces más que satisfacer las necesidades de salud, del individuo las empeoran o agravan.

Para tener una idea más amplia de la situación problemática citaremos a Schar en Alemania, que halló que la automedicación se practica entre el $60 \%$ y $80 \%$ de los casos con algún problema de salud (3). Otros estudios realizados muestran la magnitud del problema como en Colombia, el estudio efectuado por
Buritica O. y otros autores, señalaron que el $47 \%$ de los estudiantes de la Universidad de Caldas se automedican, y la facultad donde más se automedica fue en Ciencias de la Salud con $30.3 \%$, siendo las razones por cefalea y otros dolores (4). En Filipinas, Hardon encontró que el $80 \%$ de los menores fue tratado en sus casas, por enfermedades comunes (diarrea, tos, resfrío común), con medicamentos dañinos, contraindicados 0 combinaciones irracionales (5).

Estudios realizados en América Latina, eran demostrados que esta práctica es muy frecuente en México se encontró un resultado destacable en el cual el $80 \%$ de los productos expendidos en farmacias eran por automedicación (6). En nuestro país, en un estudio realizado por Rondinel sobre las características de consumo de medicamentos en Lima y en Yauri (Cuzco) se halló que el $32 \%$ de los medicamentos fue vendido sin prescripción médica, siendo mayor en el estrato socioeconómico alto (7). En Ica, un estudio realizado por Bendezú et al, concluyó que hay una prevalencia del $72,9 \%$ de automedicación entre los alumnos.

Dentro de la automedicación, los fármacos más utilizados pertenecen al grupo de los antibióticos y AINES(9). Estas tasas de automedicación son preocupantes puesto que las consecuencias pueden ser importantes como enmascarante de la enfermedad, 
aparición de efectos adversos, prolongación o resistencia a los medicamentos empleados, facilitar el contagio y fomento de la drogo dependencia (10). Partiendo de la hipótesis de que el comportamiento de los estudiantes universitarios frente a la automedicación estaría encuadrado con ciertas variaciones en el lineamiento de conducta de la población en general, y al no existir estudios que indiquen las características específicas de la automedicación en poblaciones bien definidas como la de los estudiantes universitarios, siendo con frecuencia este grupo poblacional expuesto a la automedicación.

Por todo lo expuesto, el presente estudio se realizó con el objetivo de determinar los factores condicionantes de la automedicación en estudiantes de la Facultad de Enfermería de la Universidad Nacional San Luis Gonzaga de Ica.

\section{MATERIAL Y MÉTODOS}

En este trabajo de investigación se utilizó el método descriptivo de corte transversal. La muestra estuvo conformada por 136 estudiantes del I al IX Ciclo, obteniéndose esta muestra aplicando una fórmula para población finita. Se excluyeron estudiantes que se encuentran realizando prácticas preprofesionales (internado, en zonas periféricas de Ica y fuera de ella). Se hizo uso de un cuestionario con 13 reactivos de opción múltiple.

En la recolección de datos se utilizó como técnica la encuesta y como instrumento el cuestionario elaborado por los autores previamente validado. Para evaluar la confiabilidad del instrumento se realizó una prueba piloto al $15 \%$ de la muestra en estudio (no se incluye los 136 estudiantes), que permitió determinar las características finales del instrumento. Haciendo uso del coeficiente de confiabilidad Alfa Cronbach, obteniéndose un valor de 0,75. Así como la prueba estadística de chi cuadrado $\left(x^{2}\right)$ para determinar la significancia o no entre los indicadores y los ciclos de estudios. Se utilizaron los programas de análisis de datos tabulados EPIDAT 2.1 y SPSS.

\section{RESULTADOS}

El $90,4 \%$ de los estudiantes de la Facultad de Enfermería son del sexo femenino y se da en todos los ciclos. Entre los factores que condicionan la automedicación señalaron dentro del entorno social farmacéutico y la familia $52,9 \%(72)$ y $30,9 \%$ (42) $p<0,01$. Factores culturales: $39 \%$ (53 estudiantes), visitaron al médico cuando están enfermos $(P>0,05)$. Siendo el medio de comunicación más influenciable la televisión 74,3\% (101 estudiantes) ( $P>0,05)$. Factores económicos. El lugar de compra del medicamento fue la farmacia $89 \%$ (121), desestimándose el $52,2 \%$ (71 estudiantes) dentro del ingreso económico familiar para acceder a un servicio de salud (Tabla 1).

El 33,8\% (46 estudiantes) $P>0,05$ se automedica cuando tienen dolor de cabeza, fiebre y cólicos menstruales $(39,36$ y $31 \%$ respectivamente) $\mathrm{P}>0,05$. Los medicamentos que se ingieren frecuentemente en los últimos 06 meses sin prescripción médica son: analgésicos/antiinflamatorios $75,7 \% \quad$ (103), antibióticos $11,8 \%$ (16), antigripales 8,1\% (11) (Tabla 2).

TABLA N 01

FACTORES QUE CONDICIONAN LA AUTOMEDICACIÓN

\begin{tabular}{lrr}
\hline FACTORES & N & $(\%)$ \\
\hline Sexo & & \\
Masculino & 13 & 9,6 \\
Femenino & 123 & 90,4
\end{tabular}

$\begin{array}{lrr}\text { A. FACTORES SOCIALES } & & \\ \text { Quien recomendó el } & 42 & 30,9 \\ \text { medicamento } & 4 & 2,9 \\ \text { Familia } & 72 & 52,9 \\ \text { Amigos } & 10 & 7,4 \\ \text { Farmacéutico } & 8 & 5,9 \\ \text { Medios de comunicación } & & \\ \text { Otros } & & \end{array}$




\section{B. FACTORES CULTURALES}

Nivel de conocimiento

Automedicación es:

Uso de medicamentos sin 112 82,4

prescripción médica

Tomar medicamentos sin $9 \quad 6,6$

conocer sus reacciones

Ingerir medicamentos para $7 \quad 5,1$

calmar sus síntomas

Consumir medicamentos

anteriormente recetados

Comprar medicamentos con $\begin{array}{lll}6 & 4,4\end{array}$

receta médica

Considera que tiene efectos para la salud.

Buenos para la salud

Malos para la salud

Buenos para la economía

No tiene ningún efecto

Visita al médico cuando está enfermo

$\mathrm{Si}$

No

$A$ veces

Medios de comunicación que influyen

Televisión

Radio

Anuncios

Revistas

Otros

\section{FACTORES ECONÓMICOS}

Donde obtuvo el medicamento:

Farmacia

Establecimiento de salud

Tienda

Se la regalaron

\section{Dinero destinado para acceder} a un servicio de salud.

$\mathrm{Si}$

No

$A$ veces
10174,3

$1 \quad 0,7$

$14 \quad 10,3$

$7 \quad 5,1$

137,6
TABLA N ${ }^{\circ} 02$

CARACTERÍSTICAS DE LA

AUTOMEDICACIÓN

\begin{tabular}{lrr}
\hline CARACTERÍSTICAS & N & ( \%) \\
\hline Se automedica para & & \\
solucionar un problema de & & \\
dolencia & 46 & 33,8 \\
Si & 29 & 31,3 \\
No & 61 & 44,9 \\
A veces & & \\
Motivo de Automedicación & & \\
Fiebre & 36 & 26,5 \\
Diarrea & 4 & 2,94 \\
Dolor de garganta & 15 & 11,0 \\
Dolor de cabeza & 39 & 28,7 \\
Malestar general & 10 & 7,4 \\
Cólicos menstruales & 31 & 22,8 \\
Insomnio & 1 & 0,7 \\
MEDICAMENTOS MÁS & & \\
UTILIZADOS EN LOS & & \\
ÚLTIMOS 06 MESES & & \\
Analgésicos / Antiinflamatorios & 103 & 75,7 \\
Antigripales & 11 & 8,1 \\
Anticonceptivos & 5 & 3,7 \\
Antibióticos & 16 & 11,8 \\
Ninguno & 1 & 0,7 \\
\hline
\end{tabular}

\section{DISCUSIÓN}

Los estudiantes de enfermería son en su mayoría mujeres, razón por la cual el estudio mostró que el $90,4 \%$ de sexo femenino coincidente con el hallazgo de Aguado en estudiantes de Farmacia de la Universidad Nacional Noreste Argentina, donde se encontró que el $67 \%$ era de sexo femenino (11).

Con respecto a los factores que condicionan la automedicación referente al entorno social, predominaron en porcentajes mayoritarios la recomendación dada por el farmacéutico y la familia $(52,5 \%$ y $30,9 \%)$, estos resultados coinciden aunque con porcentajes más elevados, en el estudio efectuado por Morillo et al en Ecuador el año 2010 (12). De lo expuesto, se señala que los resultados están relacionados a que los estudiantes confían más en el consejo directo de un tercero para la recomendación de un medicamento. 
Teniendo como base el hallazgo en la literatura que específica que la presión de grupo o de nuestros propios familiares los cuales nos ofrecen una alternativa para la solución de nuestros problemas de salud basados en su propia experiencia, los cuales ignoran por completo el riesgo que implica la automedicación (13).

Referente a factores culturales: un $61 \%$ no visita al médico o visita a veces, este resultado evidencia un peligro latente conducente al uso indiscriminado de medicamentos sin prescripción médica, tendiente a lo largo de desarrollar dependencia a determinada droga o de desencadenar los efectos adversos 0 colaterales que ello implica. La amplia mayoría de los estudiantes manifestó no desconocer los riesgos de consumir fármacos sin control médico, factor que pareció no ser tomado en cuenta a la hora de automedicarse. Aumentando las tasas de prevalencia de automedicación en nuestro medio influenciado a través de Spot publicitario de medicamentos de venta libre, evidenciado en el estudio que mostró que la televisión es el medio más influenciable $(72,8 \%)$. Sin embargo se pudo apreciar porcentajes más bajos de automedicación en este factor el $38,65 \%$ en el estudio realizado por Trillo el año 2005, donde encontró que se había comprado el medicamento luego de haberlo visto por la televisión o escuchado por la radio (14). Cifras casi similares se encontraron en Ecuador en el estudio ejecutado por Morillo $\mathrm{M}$ et al, en el cual el $44 \%$ fueron influenciados por los medios de comunicación (12).

Asimismo observamos en porcentajes mínimos en Argentina el año 2004 por Aguado et al con un 3\% que eligieron el medicamento a utilizar guiándose por los avisos publicitarios, esto sin embargo puede ser una forma pasiva pero peligrosa de fomentar el consumo y no debe ser descuidado (11).
Factor económico: Concerniente al lugar donde adquirieron el medicamento, mayoritariamente lo obtuvieron en la Farmacia (89\%) asociados a un $47,8 \%$ que no o a veces destinaban el dinero del ingreso económico familiar para acceder a un servicio de salud. Estos porcentajes son consistentes con Morillo con un $61 \%$ en ambos indicadores (12). Este resultado va permitir por un lado al profesional farmacéutico ofrezca asesoría para el uso de medicinas de venta libre y crear conciencia entre los estudiantes de la existencia de medicamentos que pueden ser adquiridos sin receta médica, pero que no están exentos de presentar reacciones o efectos colaterales. Siendo este resultado desventajoso porque queda un espacio al libre albedrio de fomento de la automedicación influenciado por el entorno familiar o publicitario.

Con respecto a las características de la automedicación, el presente estudio señala que se automedica el $33,8 \%$ de los estudiantes, siendo el motivo por dolor de cabeza $(28,7 \%)$ por fiebre $(26,5 \%)$ y por dolores menstruales (22,8\%) utilizándose analgésicos y antiinflamatorios $(75,7 \%)$ para solucionar el problema de dolencias. Buritica mostró cifras coincidentes en estudiantes colombianos que demuestran que la facultad donde más se automedicaba fue en la de Ciencias de la Salud, con 30,3\%. Los medicamentos más utilizados fueron: Antiinflamatorios no esteroides (67\%). El motivo más frecuente fue la cefalea $(33 \%)$ seguida de otros dolores $(22,4 \%)(4)$. Encontrándose un porcentaje un tanto mayor (44\%) en la población de Lambayeque, Perú señalado por Tello (14). Pero Bendezú muestra porcentajes mayoritarios a los resultados de esta investigación realizada en Ica, con un $72,9 \%$ de automedicación entre los estudiantes de la Universidad Nacional San Luis Gonzaga de Ica (UNICA) siendo aún mayor en Ciencias de la Salud que alcanzaron $82,5 \%$ el año 2004. Estos resultados revelan que en nuestro medio es 
más fácil comprar un analgésico en cualquiera de los establecimientos y lo que es más usual son los propios farmacéuticos o personal de boticas los que aconsejan el medicamento.

Situación que refuerza la necesidad de intervención en la limitación de la oferta, regulación y control por parte del Estado, como una manera de prevenir situaciones de uso inadecuado o de abuso de medicamentos sin prescripción médica. Sin embargo, Morillo en Ecuador, señala cifras más elevadas $88 \%$ de estudiantes de enfermería se automedican siendo este un padecimiento muy común a nivel mundial sobre todo cuando se habla de la utilización de medicamentos de venta libre que están disponibles al público sin la necesidad de una prescripción médica (12).

\section{CONCLUSIONES}

Los hallazgos obtenidos en el presente estudio sobre automedicación fueron $33,8 \%$, inferiores a otros estudios a nivel local (Ica) 72,9\%(8), nacional (Lambayeque) 44\% (14), internacional (Ecuador 88\% (12) y Argentina $85 \%$, etc) (11), es una cifra significativa que se tiene que considerar ya que la automedicación es un problema de Salud Pública a nivel mundial, situación que tiene que limitarse y crear conciencia en los protagonistas que ponen en peligro de riesgo de muerte la vida por los efectos adversos que puede desencadenar el fármaco y que son los profesionales de Salud (médicos, enfermeras y farmacéuticas que tienen el deber de informar, educar acerca de esta práctica y aconsejar la manera más adecuada de realizar una automedicación responsable en el uso de medicamentos de ventas libre o evitarla.

\section{Correspondencia:}

Dra. Isabel N. Urure Velazco

Correo electrónico: isabelnauv@yahoo.com

\section{REFERENCIAS BIBLIOGRÁFICAS}

1. World Health Organization. The Role of the pharmacist in self-care and selfmedication. Ginebra: WHO; 1996.

2. Lalama M. Perfil de consumo de medicamentos en la ciudad de Quito. Educ Med Contin. 1999; 64: 7-9.

3. Beske F, Hampft R. Status of selfmedication in West Germany. Soz Präventivmed. 1996; 31(3): 166-169.

4. Buritica O, Giraldo A, Franco D, Cañas A, Giraldo J. Automedicación en estudiantes de la Universidad de Caldas. Revista de Ciencias Básicas. 2003; 2:7-14.

5. Hardon AP. The use of modern pharmaceuticals in a Filipino Village: Doctors' prescriptions and selfmedication. Social Science and Medicine. 1991; 25:277-92.

6. Lomeli A. Graves daños a la salud. Boletín electrónico Latinoamericano para fomentar el uso adecuado de medicamentos. 2001; 4(3): 4-5.

7. Rondinel C. Características del consumo de medicamentos en Lima y Yauri (Cuzco). Tesis de Bachiller de Medicina. Lima, Perú. Universidad Peruana Cayetano Heredia, 1985.

8. Bendezú R, Franco E, Peña J, Torres E. Prevalencia de automedicación en estudiantes de la Universidad Nacional San Luis Gonzaga de Ica. Ica, Perú: II Congreso de investigación, Visión compartida de las líneas de investigación Científica; 2004.

9. Lopez F. Los peligros de la automedicación (Internet). (Citado en diciembre 2012) Disponile en: www.lospeligrosdelaautomedicación.htm

10. Llavina N. Automedicarse: consumir de forma responsable los medicamentos. Eroski Consumer (Internet); 2011. (Citado en diciembre 2011). Disponible en: http://www.consumer.es/web/es/salud/pre vencion/2011/08/29/202640.php 
11. Aguado M, Nuñez Mb, Dos Santos Antola L, Bregni C. Automedicación en Estudiantes de Farmacia de la Universidad Nacional del Nordeste, Argentina Acta Farm Bonaerense. 2005; 24:271-6.

12. Imbacuan A, Morillo M, Valencia V. Factores relacionados con la automedicación en los estudiantes de Enfermería de la Facultad de Ciencias de la Salud de la Universidad Técnica del Norte en la ciudad de Ibarra en el Periodo enero-agosto 2010. Ecuador: Universidad Técnica del Norte; 2010.
13. Alvarado S. Automedicación, Sinfonet nuestra Net y Cruz. Blanca Panameña a la comunidad; 1997.

14. Tello S, Yovera A. Factores asociados a la incidencia de la automedicación. Tesis para grado de bachiller. Chiclayo, Lambayeque, Perú. Universidad Pedro Ruiz Gallo, 2005.

Recibido: 06/11/12

Aprobado para publicación: 20/11/12 